\title{
Integrated analysis of immune-related long noncoding RNAs as diagnostic biomarkers in psoriasis
}

\author{
Fan Feixiang Equal first author, 1, 2 , Zhen Huang ${ }^{\text {Equal first author, } 2 \text {, Yongfeng Chen }}{ }^{\text {Corresp. } 1}$ \\ ${ }^{1}$ Department of Dermatology, Dermatology Hospital, Southern Medical University, Guangzhou, Guangdong, China \\ 2 Department of Dermatology, Shenzhen Longhua District Central Hospital, Shenzhen, Guangdong, China \\ Corresponding Author: Yongfeng Chen \\ Email address: gdcyf@163.com
}

Abstract Background: Psoriasis is a chronic immune-mediated inflammatory dermatosis. Long noncoding RNAs (IncRNAs) play an important role in immune-related diseases. This study aimed to identify potential immune-related IncRNA biomarkers for psoriasis.

Methods: We screened differentially expressed immune-related IncRNAs biomarkers using GSE13355 (skin biopsy samples of 180 cases) from Gene Expression Omnibus (GEO). Moreover, Gene Ontology (GO), Kyoto Encyclopedia of Genes and Genomes (KEGG), and gene set enrichment analysis (GSEA) were performed to explore biological mechanisms in psoriasis. In addition, we performed LASSO logistic regression to identify potential diagnostic IncRNAs and further verify the diagnostic value and relationship with drug response using two validation sets GSE30999 (skin biopsy samples of 170 cases) and GSE106992 (skin biopsy samples of 192 cases). Furthermore, we estimated the degree of infiltrated immune cells and investigated the correlation between infiltrated immune cells and diagnostic IncRNA biomarkers. Results: A total of 394 differentially expressed genes (DEGs) were extracted from gene expression profile. GO and KEGG analysis of target genes found that immune-related IncRNAs were primarily associated with epidermis development, skin development, collagen-containing extracellular matrix, and glycosaminoglycan binding and mainly enriched in cytokine-cytokine receptor interaction and influenza A and chemokine signaling pathway. We found that LINC01137, LINC01215, MAPKAPK5-AS1, TPT1-AS1, CARMN, CCDC18-AS1, EPB41L4A-AS, and LINC01214 exhibited well diagnostic efficacy. The ROC and ROC Cl were 0.944 (0.907-0.982), 0.953

(0.919-0.987), 0.822 (0.758-0.887), 0.854 (0.797-0.911), 0.957(0.929-0.985), 0.894 (0.846-0.942), and 0.964 (0.937-0.991) for LINC01137, LINC01215, MAPKAPK5-AS1, TPT1AS1,CARMN, CCDC18-AS1, EPB41L4A-AS1, and LINC01214. LINC01137, LINC01215, and LINC01214 were correlated with drug response. LINC01137, CCDC18-AS1, and CARMN were positively correlated with activated memory CD4 T cell, activated myeloid dendritic cell (DC), neutrophils, macrophage M1, and T follicular helper (Tfh) cells, while negatively 
correlated with T regulatory cell (Treg). LINC01215, MAPKAPK5-AS1, TPT1-AS1, EPB41L4AAS, and LINC01214 were negatively correlated with activated memory CD4 T cell, activated myeloid DC, neutrophils, macrophage $\mathrm{M1}$, and Tfh, while positively correlated with Treg. Conclusions: These findings indicated that these immune-related IncRNAs may be used as potential diagnostic and predictive biomarkers for psoriasis. 
1 Integrated analysis of immune-related long noncoding

2 RNAs as diagnostic biomarkers in psoriasis

3 Feixiang Fan ${ }^{1,2}$, Zhen Huang ${ }^{2}$, Yongfeng Chen ${ }^{1}$

4

5 6

${ }^{1}$ Department of Dermatology, Dermatology Hospital, Southern Medical University, Guangzhou, Guangdong, China

${ }^{2}$ Department of Dermatology, Shenzhen Longhua District Central Hospital, Shenzhen, Guangdong, China

Corresponding Author:

Yongfeng Chen

Lujing Road, No. 2, Guangzhou, Guangdong, 510091, China

Email address: gdcyf@163.com

\section{Abstract}

Background: Psoriasis is a chronic immune-mediated inflammatory dermatosis. Long noncoding RNAs (lncRNAs) play an important role in immune-related diseases. This study aimed to identify potential immune-related lncRNA biomarkers for psoriasis.

Methods: We screened differentially expressed immune-related lncRNAs biomarkers using GSE13355 (skin biopsy samples of 180 cases) from Gene Expression Omnibus (GEO).

Moreover, Gene Ontology (GO), Kyoto Encyclopedia of Genes and Genomes (KEGG), and gene set enrichment analysis (GSEA) were performed to explore biological mechanisms in psoriasis. In addition, we performed LASSO logistic regression to identify potential diagnostic IncRNAs and further verify the diagnostic value and relationship with drug response using two validation sets GSE30999 (skin biopsy samples of 170 cases) and GSE106992 (skin biopsy samples of 192 cases). Furthermore, we estimated the degree of infiltrated immune cells and investigated the correlation between infiltrated immune cells and diagnostic lncRNA biomarkers.

Results: A total of 394 differentially expressed genes (DEGs) were extracted from gene expression profile. GO and KEGG analysis of target genes found that immune-related lncRNAs were primarily associated with epidermis development, skin development, collagen-containing extracellular matrix, and glycosaminoglycan binding and mainly enriched in cytokine-cytokine receptor interaction and influenza $\mathrm{A}$ and chemokine signaling pathway. We found that LINC01137, LINC01215, MAPKAPK5-AS1, TPT1-AS1, CARMN, CCDC18-AS1, EPB41L4A-AS, and LINC01214 exhibited well diagnostic efficacy. The ROC and ROC CI were 0.944 (0.907-0.982), $0.953(0.919-0.987), 0.822$ (0.758-0.887), 0.854 (0.797-0.911), 0.957(0.929-0.985), 0.894 (0.846-0.942), and 0.964 (0.937-0.991) for LINC01137, LINC01215, MAPKAPK5-AS1, TPT1-AS1,CARMN, CCDC18-AS1, EPB41L4A-AS1, and LINC01214. LINC01137, LINC01215, and LINC01214 were correlated with drug response. LINC01137, CCDC18-AS1, and CARMN were positively correlated with activated memory 
40 CD4 T cell, activated myeloid dendritic cell (DC), neutrophils, macrophage M1, and T follicular

41 helper (Tfh) cells, while negatively correlated with T regulatory cell (Treg). LINC01215,

42 MAPKAPK5-AS1, TPT1-AS1, EPB41L4A-AS, and LINC01214 were negatively correlated

43 with activated memory CD4 T cell, activated myeloid DC, neutrophils, macrophage M1, and

44 Tfh, while positively correlated with Treg.

45 Conclusions: These findings indicated that these immune-related lncRNAs may be used as

46 potential diagnostic and predictive biomarkers for psoriasis.

47

\section{Introduction}

49 Psoriasis is a chronic immune-mediated inflammatory dermatosis that affects $0.09 \%-5.1 \%$ of people worldwide, with incidence increasing annually (Boehncke \& Schön, 2015; Michalek, Loring, \& John, 2017). The patients' quality of life is seriously affected because psoriasis is usually persistent and prone to relapse. The pathogenesis of psoriasis involves dysregulation of innate and adaptive immune system; however, the specific immunopathogenic mechanisms remain unclear (Albanesi et al., 2018). Therefore, investigation of immune-related diagnostic biomarkers and a better understanding of immunopathogenic mechanisms of psoriasis are important.

Long noncoding RNAs (lncRNAs) are transcripts longer than 200 nucleotides that generally do not code for proteins. They play pivotal roles in a number of physiological and pathological processes (Kopp \& Mendell, 2018). Recent study has identified a variety of differentially expressed lncRNAs in psoriatic lesions that were changed after biologics therapy (Gupta et al., 2016). Previous studies focused on the correlation between lncRNAs and psoriatic keratinocytes (Duan et al., 2020); several lncRNAs, including TINCR, PRANCR and ANCR, played an important role in epidermal homeostasis (Cai et al., 2020; Kretz et al., 2013; Kretz et al., 2012). Research has revealed that PRINS is involved in psoriasis pathogenesis by regulating keratinocyte stress response and apoptosis (Szell et al., 2016). however, the role of lncRNAs in the psoriasis immune abnormalities has not been reported. To date, studies have indicated that lncRNAs are involved in DC differentiation and activation of innate immune response (Wang et al., 2014; Xu et al., 2019). Moreover, lncRNAs play important role in T cell differentiation and immune-related diseases (Roy \& Awasthi, 2019). They exhibit cell- and tissue-specific expression (Liu et al., 2017; Tsoi et al., 2015). Given this, immune-related lncRNAs may be used as potential diagnostic and prognostic biomarkers for psoriasis. In recent years, bioinformatics analysis has provided new insight into the molecular mechanism and therapeutic targets in psoriasis (Anbunathan \& Bowcock, 2017). Weighted gene coexpression network analysis (WGCNA) has been used to identify potential biomarkers for psoriasis (Sundarrajan \& Arumugam, 2016). In our previous study, WGCNA was used to identify potential key mRNAs and lncRNAs for psoriasis (Li et al., 2020). However, immunerelated lncRNAs in the pathogenesis of psoriasis and the correlation between immune-related lncRNAs and treatment response have been relatively neglected. 
79 In this study, we screened differentially expressed genes (DEGs) and differentially expressed

80 immune-related genes (DEIRGs) from training set and identified immune-related lncRNA

81 biomarkers using coexpression analysis. Next, we validated the diagnostic efficacy of 10

82 IncRNAs and its correlation with biologics response using 2 validation sets, respectively. In

83 addition, we investigated the correlation between infiltrated immune cells and immune-related

84 IncRNAs. A total of 394 differentially expressed genes (DEGs) and 76 DEIRGs were extracted

85 from the gene expression profile. Coexpression analysis identified 16 immune-related lncRNAs.

86 Of 16 immune-related lncRNAs, 10 lncRNAs were identified as potential diagnostic biomarkers

87 for psoriasis using LASSO logistic regression algorithms.

88

\section{Materials \& Methods}

90 Gene expression data processing

91 The psoriasis gene expression profile datasets GSE13355 (Nair et al., 2009), GSE30999 (Correa

92 da Rosa et al., 2017; Suárez-Fariñas et al., 2012), and GSE106992 (Brodmerkel et al., 2019)

93 were downloaded from Gene Expression Omnibus (GEO) database

94 (https://www.ncbi.nlm.nih.gov/geo/) using the GEOquery (Davis \& Meltzer, 2007) package of R

95 software (version 3.6.5, http://r-project.org/). All samples of the datasets were derived from

96 Homo sapiens, and the platform was based on GPL570 [HG-U133_Plus_2] Affymetrix Human

97 Genome U133 Plus 2.0 Array. Affymetrix includes 47,400 probes and represents 38,500 human

98 genes. Gene biotypes were extracted using the BioMart (Durinck et al., 2009). Gene biotypes

99 were used to distinguish lncRNAs, miRNAs and mRNAs, and the expression matrix of lncRNAs

100 was extracted separately. There are 1313 lncRNAs on the Affymetrix Human Genome U133

101 Plus 2.0. GSE13355 consisted of 58 psoriasis lesion samples, adjacent normal skin samples, and

10264 normal skin samples from normal controls. GSE30999 consisted of 85 psoriasis lesion

103 samples and adjacent normal skin samples. GSE106992 consisted of 192 skin samples of

104 moderate to severe psoriasis patients undergoing ustekinumab (a monoclonal antibody directed

105 against the P40 unit of IL-12 and IL-23) or etanercept (a TNF antagonist) therapy. Patients were

106 categorized as responders and nonresponders. Responders vs nonresponders was determined

107 based on whether the PASI75 score was reached following treatment with ustekinumab or

108 etanercept for 12 weeks. All 3 datasets were included in this study. The raw data of GSE13355,

109 GSE30999, and GSE106992 datasets were read using the affy package (Gautier et al., 2004).

110 Background correction and normalization were performed, and distinguishable lncRNA and 111 mRNA gene expression matrices were obtained. z-score normalization for GSE13355 dataset

112 was performed using the limma package. The effect of correction was presented using principal

113 component analysis (PCA) using ggplot2 package (Ginestet, 2011). GSE13355 was used as

114 training set whereas GSE30999 and GSE106992 were used as validation sets. This study did not

115 involve studies on human participants or animals performed by any of the authors.

116 Screening of differentially expressed genes

117 The limma package (Ritchie et al., 2015) was used to screen GSE13355 dataset DEGs by

118 comparing lesion samples, adjacent normal skin samples, and normal controls. A cutoff value of 
119 adjusted $\mathrm{P}<.05$ and $|\log 2 \mathrm{FC}|>1$ was considered statistically different. Subsequently, volcano plot 120 was performed using ggplot2 to visualize DEGs.

121 Functional and pathway enrichment analysis of DEGs

122 Gene Ontology (GO) serves as a powerful tool to annotate genes and analyze related biological

123 processes of genes, and Kyoto Encyclopedia of Genes and Genomes (KEGG) is a bioinformatics

124 resource for understanding high-level functions and utilities of the biological system. GO and

125 KEGG analyses of DEGs were performed using clusterProfiler package (Yu et al., 2012),

126 adjusted $\mathrm{P}<.05$ was considered statistically significant. Gene set enrichment analysis (GSEA) is a

127 statistical approach for determining whether the genes from particular pathways or other

128 predefined gene sets are differentially expressed in different phenotypes (Subramanian et al.,

129 2005). Reactome pathways were analyzed with GSEA, using clusterProfiler (Yu et al., 2012) to

130 define every functional cluster. "c2.cp.kegg.v7.0.symbols.gmt" was selected as reference set, and

131 false discovery rate $(\mathrm{FDR})<0.25$ with $\mathrm{P}<.05$ was considered significantly enriched.

132 Screening of immune-related genes and immune-related IncRNAs

133 The list of immune-related genes (IRGs) was downloaded from ImmPort

134 (https://immport.niaid.nih.gov) database (Bhattacharya et al., 2018). DEIRGs from DEGs were

135 identified, and volcano plots (differential expression of DEIRGs) were plotted using ggplot2

136 package. Immune-related lncRNAs were screened using coexpression analysis of DEIRG and

137 lncRNA expression matrices. Correlation coefficients $>.4$ with $\mathrm{P}<.05$ was considered as

138 coexpression (Xiong et al., 2019; Deforges et al.,2019). Likewise, target genes were screened

139 using coexpression analysis of $\operatorname{lncRNA}$ and mRNA expression matrices.

140 GO and KEGG enrichment analyses of IRGs and immune-related IncRNAs

141 To analyze the functions of IRGs and immune-related lncRNAS, GO and KEGG enrichment

142 analyses were performed using clusterProfiler, and adjusted $\mathrm{P}<.05$ was considered statistically

143 significant.

144 Screening and validation of immune-related IncRNA biomarkers

145 Biomarkers of psoriasis were screened using LASSO logistic regression feature selection

146 algorithm (Tibshirani, 1996) based on immune-related lncRNA expression matrices. We used a

147 LASSO-logitstic-algorithm model; further, a 10-fold cross-validation was used to identify the

148 optimal lambda value. Diagnostic performances were validated using GSE30999 dataset as

149 validation sets, an AUC value $>0.7$ was determined to indicate acceptable diagnostic efficacy

150 (Watson et al.,2015; Bhardwaj et al., 2020), whereas the correlation between lncRNA

151 biomarkers and therapeutic response was validated using GSE106992 dataset.

152 Assessment of immune cell infiltration and the correlation between biomarkers and

153 immune cells

154 To estimate the composition and abundance of immune cells in the mixed cells, deconvolution of

155 transcriptome expression matrices was performed using CIBERSORT (Newman et al., 2015)

156 based on linear support vector regression. Expression matrices were uploaded to CIBERSORT,

157 and immune cell infiltration matrices were generated with cutoff value of $\mathrm{P}<.05$. Heatmap was

158 generated using R language "pheatmap" package (https://CRAN.R- 
159 project.org/package=pheatmap) to visualize 22 infiltrated immune cells in each sample. Two-

160 dimensional PCA plots were visualized using ggplots, and heatmap was plotted using the 161 corrplot package (Friendly, 2002) to visualize the correlation of 22 immune cell types. Violin 162 plots were generated using ggplot2 package to visualize the infiltration difference of 22 immune 163 cell types. Infiltrating immune cells-related network plots were generated using igraph package 164 (Ju et al., 2016) to visualize the interactions of infiltrated immune cells. $\mathrm{P}<.05$ and correlation 165 coefficients $>0.4$ were considered statistically significant. Correlation analysis was performed 166 between immune-related lncRNA biomarkers and infiltrated immune cells. Afterwards, results 167 were visualized using ggplot2 package.

168

\section{Results}

\section{Gene expression data preprocessing and DEGs identification} Figure 1 represents the study flowchart. Primarily, gene expression matrices of GSE13355 dataset were normalized. PCA was plotted before and after normalization (Figure 2A and 2B). The results indicated that sample clustering was more apparent after normalization, which indicated that the sample source was reliable. A total of 394 DEGs were extracted from gene expression profile using R software after data preprocessing, as shown in the volcano plot (Figure 2C). The details of top 10 upregulated and downregulated differently expressed genes are presented in Table 1 and Table 2. GO analysis revealed that DEGs were primarily associated with epidermis development, skin development, secretory granule lumen, and receptor ligand activity (Figure 3A). The results of KEGG analysis indicated that DEGs were mainly enriched in cytokine-cytokine receptor interaction and IL-17 signaling pathway (Figure 3B). GESA suggested that psoriasis was mainly involved in IL-17 signaling pathway and proteasome pathway (Figure 3C). CCL2, CCL7, CCL20, PSMB8, PSMB9, and PSMB10 played important roles in signal transduction of the 2 pathways. Detailed enrichment results were presented in Supplemental Table 1. Identification of IRGs and immune-related IncRNAs A total of 76 DEIRGs were extracted from gene expression profile, as shown in volcano plots and heatmap (Figure 4A and FigureS1), these data only refer to GSE13355, and detailed results of GO analysis of 76 DEIRGs are presented in Supplemental Table 2. Coexpression analysis identified 16 immune-related lncRNAs, which are part of the 394 DEGs. The detailed results of KEGG analysis of 16 immune-related lncRNAs are presented in Table 3. GO enrichment analysis of target genes found that immune-related lncRNAs were primarily associated with epidermis development, skin development, collagen-containing extracellular matrix, and glycosaminoglycan binding (Figure 4B), and KEGG enrichment analysis of target genes found that immune-related lncRNAs were mainly enriched in cytokine-cytokine receptor interaction and influenza A and chemokine signaling pathway (Figure 4C). Detailed results of immune-related lncRNAs target gene functional correlation analysis are presented in 
199 Supplemental Table 3, and detailed results of coexpression analysis are presented in

200 Supplemental Table 4.

201 Identification and validation of diagnostic biomarkers

202 Of 16 immune-related lncRNAs, 10 lncRNAs were identified as potential diagnostic biomarkers

203 for psoriasis using LASSO logistic regression algorithms (Figure 5A). Detailed LASSO results

204 are presented in Supplemental Table 5. To further assess diagnostic efficacy, we performed

205 validation using GSE30999 dataset. The ROC curve (Figure 5B and 5C) indicated that

206 LINC01137, LINC01215, MAPKAPK5-AS1, TPT1-AS1, CARMN, CCDC18-AS1,

207 EPB41L4A-AS, and LINC01214 exhibited well diagnostic efficacy (AUC $>0.7$ ), which indicated

208 high diagnostic value of the screened lncRNA biomarkers. The details of the AUC and 95\% CI

209 of AUC of the 10 immune-related lncRNAs are presented in Table 4.

210 Analysis of relation between screened biomarkers and biologics therapeutic response

211 Results of drug response (Figure 6) indicated that LINC01137, LINC01215, MAPKAPK5-AS1,

212 TPT1-AS1, CARMN, CCDC18-AS1, EPB41L4A-AS, and LINC01214 were significantly

213 expressed between the responders and nonresponders groups, which was statistically significant

214 ( $\mathrm{P}<.00001)$. The expression of LINC01137, LINC01215, and LINC01214 was higher in the

215 responders group.

216 Analysis of immune cell infiltration and correlation assessment of immune cells and

217 diagnostic biomarkers

218 Heatmap of immune cell infiltration and results of cluster PCA indicated a significant difference 219 between the psoriasis group and control group (Figure 7A and 7B). Heatmap of 22 immune cells 220 indicated that psoriasis was positively correlated with activated memory CD4+T cell, activated 221 myeloid DC, neutrophils, and T follicular helper (Tfh) cells, while negatively correlated with $\mathrm{T}$

222 regulatory cell (Tregs) and activated mast cell. Violin plots of immune cell infiltration difference 223 (Figure 8A) indicated that naïve $\mathrm{B}$ cell, $\mathrm{CD} 8+\mathrm{T}$ cell, activated memory $\mathrm{CD} 4+\mathrm{T}$ cell, Tfh cell, $\mathrm{T}$

224 gamma delta cell, NK cell resting, macrophage M0, macrophage M1, activated myeloid DC, and 225 neutrophil were higher than normal control, whereas B cell memory, B cell plasma, T cell CD4+ 226 naïve, Tregs, activated NK cell, and activated mast cell were lower. Plots of 22 immune cells 227 interaction (Figure 8B) indicated that activated mast cell exhibited the strongest interaction with 228

229

230

231

232

233

234

235 other immune cells, whereas CD8+T cell and monocyte were the weakest. Results of the correlation analysis indicated that activated memory CD4 T cell, activated myeloid DC, neutrophil, and Tfh were significantly positively correlated with LINC01137, CCDC18-AS1, and CARMN, while negatively correlated with LINC01215, MAPKAPK5-AS1, LINC01305, DUBR, TPT1-AS1, EPB41L4A-AS, and LINC01214. In addition, Tregs and activated mast cell were found to be significantly negatively correlated with LINC01137, CCDC18-AS1, and CARMN, while positively correlated with LINC01215, MAPKAPK5-AS1, LINC01305, DUBR, TPT1-AS1, EPB41L4A-AS, and LINC01214.

236

\section{Discussion}


238

239

240

241

242

243

244

245

246

247

248

249

250

251

252

253

254

255

256

257

258

259

260

261

262

263

264

265

266

267

268

269

270

271

272

273

274

275

276

277

Psoriasis is a chronic immune-mediated inflammatory dermatosis that significantly affects patients' quality of life (Alexander \& Nestle, 2017). To date, the specific pathogenesis is unclear. Crosstalk of keratinocytes and immune cells including DCs, T cells, mast cells, and neutrophils plays an important role in the pathogenesis of psoriasis, Cytokine-cytokine receptor pathway transmits intercellular interactions, with IL-23/IL-17 pathway currently being the most investigated (Chiricozzi et al., 2018). lncRNAs are closely associated with immune-related diseases (Roy \& Awasthi, 2019); however, the role of lncRNAs in psoriasis immune abnormalities remains elusive. In our study, we explored the potential biological and diagnostic efficacy of immune-related lncRNAs in psoriasis.

This study aimed to identify key immune-related lncRNAs involved in the pathogenesis of psoriasis. We performed systematic analysis of expression profile from GSE13355 dataset; 16 immune-related lncRNAs were identified using coexpression analysis for further analysis. GO analysis has found that identified lncRNAs were enriched in biological processes related to epidermis development, skin development, and collagen-containing extracellular matrix, and KEGG analysis has found that identified lncRNAs were associated with cytokine-cytokine receptor interaction and influenza $A$ and chemokine signaling pathway, consistent with the previous study (Li \& Meng, 2019). Some studies have indicated that lncRNAs can regulate the expression of cytokines and chemokines (Dong et al., 2020; Qi et al., 2020). Previous studies have indicated that cytokine-cytokine interaction and chemokine pathway play a crucial role in the pathogenesis of psoriasis (Benezeder \& Wolf, 2019). However, the role of influenza A pathway in psoriasis has not yet been reported. Therefore, based on our findings, we suggested that these identified immune-related lncRNAs may be involved in the pathogenesis of psoriasis by regulating cytokine-cytokine interaction and chemokine pathway.

Previous studies have found that some key genes or proteins may serve as potential biomarkers for psoriasis (Dand et al., 2019; Yadav, Singh, \& Singh, 2018). However, immunerelated lncRNA biomarkers for psoriasis have not yet been reported. Of the 16 immune-related lncRNAs, 10 lncRNAs were identified as potential diagnostic biomarkers for psoriasis using LASSO algorithms. To further assess the diagnostic efficacy, we performed validation using GSE30999 dataset. ROC curve indicated that LINC01137, LINC01215, MAPKAPK5-AS1, TPT1-AS1, CARMN, CCDC18-AS1, EPB41L4A-AS, and LINC01214 exhibited well diagnostic efficacy (AUC>0.7), which indicated high diagnostic value of the screened lncRNA biomarkers. Although the appearance of novel biologics such as TNF inhibitor, IL-12/23 inhibitor, IL17A/IL-17RA inhibitor, and phosphodiesterase 4 (PD4) inhibitor has improved treatment efficacy, treatments is far from optimal because of higher medical expenses or no response to biologics therapy or residual lesions after treatment or clinical recurrence (Masson Regnault et al., 2017; Sawyer et al., 2019). Therefore, early evaluation of the response to biologics treatment is important. This study also aimed to validate the correlation between immune-related lncRNAs and response to biologics therapy. Results of drug response indicated that LINC01137, LINC01215, MAPKAPK5-AS1, TPT1-AS1, CARMNCCDC18-AS1, EPB41L4A-AS, and LINC01214 were differentially expressed between the responders and nonresponders groups. 
278 This difference was statistically significant where the expression of LINC01137, LINC01215, 279 and LINC01214 was higher in the responders group. These results indicate that LINC01137, 280 LINC01215, and LINC01214 may act as potential prognostic biomarkers for monitoring 281 therapeutic response.

282 To date, there are only a few studies on these lncRNAs. LINC01137 showed upregulated 283 expression in human HepG2 cells when exposed to chemical stress (Tani et al., 2019). The 284 function of LINC01137 still remains to be determined. LINC01215 acted as a hub gene involved 285 in the rehabilitation process through the $\mathrm{T}$ cell receptor signaling pathway in respiratory 286 syncytial virus infection (Qian, Zhang, \& Wang, 2019). LINC01214 was overexpressed in non-

287

288

289

290

291

292

293

294

295

296

297

298

299

300

301

302

303

304

305

306

307

308

309

310

311

312

313

314

315

316

317 small cell lung carcinoma (Acha-Sagredo et al., 2020). However, its exact function remains unknown. As an immune-related lncRNA, MAPKAPK5-AS1 may act as prognostic biomarker for anaplastic gliomas (Wang et al., 2018). Another study found that MAPKAPK5-AS1 was significantly overexpressed in colorectal cancer and played a role by inhibiting P21 expression (Ji et al., 2019). Its immunologic mechanisms have not yet been reported. In addition, involvement of CARMN, TPT1-AS1, and EPB41L4A-AS in cancer pathogenesis were reported (Jiang et al., 2018; Kouhsar et al., 2019; Roychowdhury et al., 2020). There has been no report of CCDC18-AS1 in the literature.

To further validate the correlation between immune-related lncRNAs diagnostic biomarkers and infiltrating immune cells, CIBERSORT was applied to estimate the infiltrating immune cells in psoriasis. In immune cell infiltration matrices, increased activated memory CD4+T cell, activated myeloid DC, neutrophil, and Tfh cell were observed, whereas Treg decreased in psoriatic lesions, consistent with previous studies. Psoriasis is an immune-driven dermatosis (Benhadou, Mintoff, \& Del Marmol, 2019). The IL-23/IL17 axis is the main immune pathway in the pathogenesis of psoriasis, and the main immune cells involved in psoriasis include CD4+T cells, DCs, neutrophils, macrophages, and Tfh (Chiricozzi et al., 2018). Tregs are important in suppressing the immune response, whereas they decrease in psoriatic lesions (Yang et al., 2016). Of the LINC01137, CCDC18-AS1, CARMN, LINC01215, MAPKAPK5-AS1, TPT1-AS1, EPB41L4A-AS, and LINC01214, LINC01137, CCDC18-AS1, and CARMN were positively correlated with activated memory CD4 T cell, activated myeloid DC, neutrophil, macrophage M1, and Tfh, while negatively correlated with Treg. In addition, LINC01215, MAPKAPK5AS1, TPT1-AS1, EPB41L4A-AS, and LINC01214 were negatively correlated with activated memory CD4 T cell, activated myeloid DC, neutrophil, macrophage M1, and Tfh, while positively correlated with Treg. These correlations may partly be explained by LINC01137, CCDC18-AS1, and CARMN that induce activation of CD4+T cells, myeloid DCs, neutrophils, macrophages, and Tfh cells and exhibit Treg cells involved in the immunopathogenesis of psoriasis, whereas LINC01215, MAPKAPK5-AS1, TPT1-AS1, EPB41L4A-AS, and LINC01214 work in the opposite way.

Our findings suggest that LINC01137, LINC01215, MAPKAPK5-AS1, TPT1-AS1, CARMN, CCDC18-AS1, EPB41L4A-AS, and LINC01214 may be potential diagnostic biomarkers for psoriasis and LINC01137, LINC01215, and LINC01214 may serve as predictive 
318 biomarkers for biologics response in psoriasis. These immune-related lncRNAs may involve in 319 the immunopathogenesis of psoriasis by activating or inhibiting related immune cells.

320

321

322

323

324

325

326

327

328

329

330

331

332

333

334

335

336

337

338

339

340

341

342

343

344

345

346

347

348

349

350

351

352

353

354

355

356

357

In the past few years, psoriasis genome-wide association studies (GWAS) have been conducted worldwide, and numbers of genetic loci associated with psoriasis susceptibility have been estimated (Ogawa \& Okada, 2020). Previews studies showed that approximately $10 \%$ of autoimmune disease-associated SNPs localize to lncRNA genes present in autoimmune diseaseassociated loci, and SNPs can affect the expression of lncRNAs (Kumar et al., 2013). Therefore, as a future prospect, identifying whether these estimated immune-related lncRNAs biomarkers contain psoriasis-related SNPs identified by GWAS will be worthwhile.

This study has some limitations. First, these results were generated by bioinformatics analysis and need further experimental verification. Second, a larger sample size of the dataset is needed for internal validation. Third, additional clinical information is needed to explore the correlation between immune-related lncRNAs and clinical severity. Fourth, Th17 is a CD4+T cell subtype; further, because the 22 immune-infiltrating cells estimated by CIBERSORT were not specific to the Th17 subgroup, Th17 cells were not observed in immune cell infiltration. Finally, the normal control group needs to be involved to analyze the differentially expressed immune-related lncRNAs.

\section{Conclusions}

We found that LINC01137, LINC01215, MAPKAPK5-AS1, TPT1-AS1, CARMN, CCDC18AS1, EPB41L4A-AS, and LINC01214 may be potential diagnostic biomarkers for psoriasis. LINC01137, LINC01215, and LINC01214 may serve as predictive biomarkers for biological response in psoriasis.

\section{Acknowledgments}

The authors would like to thank Enago (www.enago.cn) for the English language review. We sincerely thank Xuemei Wang for the technical support and useful suggestions.

\section{References}

Acha-Sagredo A, Uko B, Pantazi P, Bediaga NG, Moschandrea C, Rainbow L, Liloglou T. 2020. Long non-coding RNA dysregulation is a frequent event in non-small cell lung carcinoma pathogenesis. British Journal of Cancer 122(7):1050-1058 DOI 10.1038/s41416-020-07 42-9.

Albanesi C, Madonna S, Gisondi P, Girolomoni, G. 2018. The interplay between keratinocytes and immune cells in the pathogenesis of psoriasis. Frontiers in Immunology 9:1549. DOI 10.3389/fimmu.2018.01549.

Alexander H, Nestle FO. 2017. Pathogenesis and immunotherapy in cutaneous psoriasis: what ca $\mathrm{n}$ rheumatologists learn? Current Opinion in Rheumatology 29(1):71-78 DOI 10.1097/B OR.0000000000000358.

Anbunathan H, Bowcock AM. 2017. The Molecular Revolution in Cutaneous Biology: The Era

Peer) reviewing PDF | (2020:06:50528:3:0:NEW 1 Feb 2021) 
358

359

360

361

362

363

364

365

366

367

368

369

370

371

372

373

374

375

376

377

378

379

380

381

382

383

384

385

386

387

388

389

390

391

392

393

394

395

396

397

of Genome-Wide Association Studies and Statistical, Big Data, and Computational Topics. Journal of InvestigativeDe rmatology 137(5):e113-e118. DOI 10.1016/j.jid.2016.03.047.

Benezeder T, Wolf P. 2019. Resolution of plaque-type psoriasis: what is left behind (and reinitiates the disease). Seminars in Immunopathology 41(6):633-644 DOI 10.1007/s00281-019-00766-z.

Benhadou F, Mintoff D, Del Marmol V. 2019. Psoriasis: Keratinocytes or Immune Cells - Which Is the Trigger? Dermatology, 235(2):91-100 DOI 10.1159/000495291.

Bhardwaj M, Weigl K, Tikk K, Benner A, Schrotz-King P, Brenner H. 2020. Multiplex screening of 275 plasma protein biomarkers to identify a signature for early detection of colorectal cancer. Molecular Oncology 14(1):8-21 DOI 10.1002/1878-0261.12591.

Bhattacharya S, Dunn P, Thomas CG, Smith B, Schaefer H, Chen J, Butte AJ. 2018. ImmPort, to ward repurposing of open access immunological assay data for translational and clinical $\mathrm{r}$ esearch. Scientific Data 5(1):180015-180015 DOI 10.1038/sdata.2018.15.

Boehncke, WH, Schön MP. 2015. Psoriasis. The Lancet, 386(9997), 983-994. DOI 10.1016/s01 40-6736(14)61909-7.

Brodmerkel C, Li K, Garcet, S, Hayden K, Chiricozzi A, Novitskaya I, Krueger JG. 2019. Modulation of inflammatory gene transcripts in psoriasis vulgaris: Differences between ustekinumab and etanercept. Journal of Allergy and Clinical Immunology 143(5):19651969 DOI 10.1016/j.jaci.2019.01.017.

Cai P, Otten ABC, Cheng B, Ishii MA, Zhang W, Huang B, Sun BK. 2020. A genome-wide long noncoding RNA CRISPRi screen identifies PRANCR as a novel regulator of epidermal homeostasis. Genome Research 30(1):22-34 DOI 10.1101/gr.251561.119.

Chiricozzi A, Romanelli P, Volpe E, Borsellino G, Romanelli M. 2018. Scanning the Immunopa thogenesis of Psoriasis. International Journal of Molecular Sciences 19(1):179-210 DOI 10.3390/ijms19010179.

Correa da Rosa J, Kim J, Tian S, Tomalin LE., Krueger, JG, \& Suarez-Farinas M. 2017. Shrinking the psoriasis assessment gap: early gene-expression profiling accurately predicts response to long-term treatment. Journal of Investigative Dermatology 137(2):305-312 DOI 10.1016/j.jid.2016.09.015.

Dand N, Duckworth M, Baudry D, Russell A, Curtis CJ, Lee SH, Consortium P. 2019. HLA-C*0 6:02 genotype is a predictive biomarker of biologic treatment response in psoriasis. Journ al of Allergy and Clinical Immunology 143(6):2120-2130 DOI 10.1016/j.jaci.2018.11.03 8.

Davis S, Meltzer PS. 2007. GEOquery: a bridge between the Gene Expression Omnibus (GEO) and BioConductor. Bioinformatics 23(14):1846-1847 DOI 10.1093/bioinformatics/btm25 4.

Deforges J, Reis RS., Jacquet P, Sheppard S, Gadekar VP, Hart-Smith G, Tanzer A, Hofacker IL, Iseli C, Xenarios I, Poirier Y. 2019. Control of Cognate Sense mRNA Translation by cisNatural Antisense RNAs. Plant Physiology 180(1):305-322 DOI 10.1104/pp.19.00043. 
398

399

400

401

402

403

404

405

406

407

408

409

410

411

412

413

414

415

416

417

418

419

420

421

422

423

424

425

426

427

428

429

430

431

432

433

434

435

436

437

Dong X, Zhao Z, Hu Y, Lu Y, Liu P, Zhang L. 2020. LncRNA COL1A1-014 is involved in the progression of gastric cancer via regulating CXCL12-CXCR4 axis. Gastric Cancer 23(2):260-272 DOI 10.1007/s10120-019-01011-0.

Duan Q, Wang G, Wang M, Chen C, Zhang M, Liu M, Zheng Y. 2020. LncRNA RP6-65G23.1 accelerates proliferation and inhibits apoptosis via p-ERK1/2/p-AKT signaling pathway on keratinocytes. Journal of Cellular Biochemistry 121(11):4580-4589 DOI 10.1002/jcb.29685.

Durinck S, Spellman PT, Birney E, Huber W. 2009. Mapping identifiers for the integration of genomic datasets with the R/Bioconductor package biomaRt. Nature Protocols 4(8): 1184-1191 DOI 10.1038/nprot.2009.97.

Friendly M. 2002. Corrgrams: exploratory displays for correlation matrices. American Statistician 56(4):316-324 DOI 10.2307/3087354.

Gautier L, Cope L, Bolstad BM, Irizarry RA. 2004. affy--analysis of Affymetrix GeneChip data at the probe level. Bioinformatics, 20(3):307-315 DOI 10.1093/bioinformatics/btg405.

Ginestet C. 2011. ggplot2: Elegant Graphics for Data Analysis. Journal of the Royal Statistical Society 174(1):245-246. DOI 10.1111/j.1467-985X.2010.00676_9.x.

Gupta R, Ahn R, Lai K, Mullins E, Debbaneh M, Dimon M, Liao W. 2016. Landscape of long noncoding RNAs in psoriatic and healthy skin. Journal of Investigative Dermatology 136(3):603-609 DOI 10.1016/j.jid.2015.12.009.

Ji H, Hui B, Wang J, Zhu Y, Tang L, Peng P, Wang K. 2019. Long noncoding RNA MAPKAPK5-AS1 promotes colorectal cancer proliferation by partly silencing p21 expression. Cancer Science 110(1):72-85 DOI 10.1111/cas.13838.

Jiang H, Huang G, Zhao N, Zhang T, Jiang M, He Y, Jiang X. 2018. Long non-coding RNA TPT 1-AS1 promotes cell growth and metastasis in cervical cancer via acting AS a sponge for miR-324-5p. Journal of Experimental and Clinical Cancer Research 37(1):169-181 DOI 10.1186/s13046-018-0846-8.

Ju W, Li J, Yu W, Zhang R. 2016) iGraph: an incremental data processing system for dynamic graph. Frontiers of Computer Science, 10(3):462-476 DOI 10.1007/s11704-016-5485-7.

Kopp F, Mendell JT. 2018. Functional classification and experimental dissection of long noncoding RNAs. Cell 172(3):393-407 DOI 10.1016/j.cell.2018.01.011.

Kouhsar M, Azimzadeh Jamalkandi S, Moeini A, Masoudi-Nejad A. 2019. Detection of novel bi omarkers for early detection of Non-Muscle-Invasive Bladder Cancer using Competing E ndogenous RNA network analysis. Science Reports 9(1):8434-8449 DOI 10.1038/s41598 -019-44944-3.

Kretz M, Siprashvili Z, Chu C, Webster DE, Zehnder A, Qu K, Khavari PA. 2013. Control of so matic tissue differentiation by the long non-coding RNA TINCR. Nature 493(7431):231235 DOI 10.1038/nature11661.

Kretz M, Webster DE, Flockhart RJ, Lee CS, Zehnder A, Lopez-Pajares V, Khavari PA. 2012. S uppression of progenitor differentiation requires the long noncoding RNA ANCR. Genes and Development 26(4):338-343 DOI 10.1101/gad.182121.111.

Peerj reviewing PDF | (2020:06:50528:3:0:NEW 1 Feb 2021) 
438

439

440

441

442

443

444

445

446

447

448

449

450

451

452

453

454

455

456

457

458

459

460

461

462

463

464

465

466

467

468

469

470

471

472

473

474

475

476

477

Kumar V, Westra HJ, Karjalainen J, Zhernakova DV, Esko T, Hrdlickova B, Wijmenga C. 2013. Human disease-associated genetic variation impacts large intergenic non-coding RNA ex pression. PLoS Genetics 9(1): e1003201 DOI 10.1371/journal.pgen.1003201.

Li H, Yang C, Zhang J, Zhong W, Zhu L, Chen Y. 2020. Identification of potential key mRNAs and LncRNAs for psoriasis by bioinformatic analysis using weighted gene co-expression network analysis. Molecular Genetics and Genomics 295(3):741-749 DOI 10.1007/s0043 8-020-01654-0.

Li X, Meng Y. 2019. Survival analysis of immune-related lncRNA in low-grade glioma. $B M C$ Cancer 19(1):813-819 DOI 10.1186/s12885-019-6032-3.

Liu SJ, Horlbeck MA, Cho SW, Birk HS, Malatesta M, He D, Attenello FJ, Villalta JE, Cho MY, Chen Y, Mandegar MA, Olvera MP, Gilbert LA, Conklin BR, Chang HY, Weissman JS, Lim DA. 2017. CRISPRi-based genome-scale identification of functional long noncoding RNA loci in human cells. Science, 355(6230):39-55 DOI 10.1126/science.aah7111.

Masson Regnault M, Konstantinou MP, Khemis A, Poulin Y, Bourcier M, Amelot F, Paul C. 2017. Early relapse of psoriasis after stopping brodalumab: a retrospective cohort study in 77 patients. Journal of the European Academy of Dermatology and Venereology 31(9): 1491-1496 DOI 10.1111/jdv.14387.

Michalek IM, Loring B, John SM. 2017. A systematic review of worldwide epidemiology of psoriasis. Journal of the European Academy of Dermatology and Venereology, 31(2), 205-212. DOI 10.1111/jdv.13854.

Nair RP, Duffin KC, Helms C, Ding J, Stuart PE, Goldgar D. 2009. Genome-wide scan reveals association of psoriasis with IL-23 and NF-kappaB pathways. Nature Genetics 41(2): 199-204 DOI 10.1038/ng.311.

Newman AM, Liu CL, Green MR, Gentles AJ, Feng W, Xu Y, Hoang CD, Diehn M, Alizadeh AA. 2015. Robust enumeration of cell subsets from tissue expression profiles. Nature Methods 15(5):453-457 DOI 10.1038/nmeth.3337.

Ogawa K, Okada Y. 2020. The current landscape of psoriasis genetics in 2020. Journal of Dermatological Science 99(1):2-8 DOI 10.1016/j.jdermsci.2020.05.008.

Qi Y, Wu H, Mai C, Lin H, Shen J, Zhang X, Xie X. 2020. LncRNA-MIAT-Mediated miR-214$3 p$ Silencing Is Responsible for IL-17 Production and Cardiac Fibrosis in Diabetic Cardio myopathy. Frontiers in Cell and Developmental Biology 8:243 DOI 10.3389/fcell.2020.0 0243.

Qian Z, Zhang Z, Wang Y. 2019. T cell receptor signaling pathway and cytokine-cytokine receptor interaction affect the rehabilitation process after respiratory syncytial virus infection. PeerJ, 7, e7089 DOI 10.7717/peerj.7089.

Ritchie ME, Phipson B, Wu D, Hu Y, Law CW, Shi W, Smyth GK. 2015. limma powers differential expression analyses for RNA-sequencing and microarray studies. Nucleic Acids Research 43(7):e47 DOI 10.1093/nar/gkv007.

Roy S, Awasthi A. 2019. Emerging roles of noncoding RNAs in T cell differentiation and functi 
478

479

480

481

482

483

484

485

486

487

488

489

490

491

492

493

494

495

496

497

498

499

500

501

502

503

504

505

506

507

508

509

510

511

512

513

514

515

516

517

ons in autoimmune diseases. International Reviews of Immunology 38(5):232-245 DOI 1 0.1080/08830185.2019.1648454.

Roychowdhury A, Samadder S, Das P, Mazumder DI, Chatterjee A, Addya S, Panda CK. 2020. Deregulation of H19 is associated with cervical carcinoma. Genomics, 112(1):961-970 DOI 10.1016/j.ygeno.2019.06.012.

Sawyer LM, Cornic L, Levin LA, Gibbons C, Moller AH, \& Jemec GB. 2019. Long-term efficacy of novel therapies in moderate-to-severe plaque psoriasis: a systematic review and network meta-analysis of PASI response. Journal of the European Academy of Dermatology and Venereology 33(2):355-366 DOI 10.1111/jdv.15277.

Suarez-Farinas M, Li K, Fuentes-Duculan J, Hayden K, Brodmerkel C, Krueger JG. 2012. Expanding the psoriasis disease profile: interrogation of the skin and serum of patients with moderate-to-severe psoriasis. Journal of Investigative Dermatology 132(11): 25522564 DOI 10.1038/jid.2012.184.

Subramanian A, Tamayo P, Mootha V, Mukherjee S, Ebert BL, Gillette MA, Paulovich A, Pomeroy SL, Golub TR, Lander ES, Mesirov JP. 2005 Gene set enrichment analysis: a knowledge-based approach for interpreting genome-wide expression profiles. Proceedings of the National Academy of Sciences USA 102:15545-15550 DOI 10.1073/pnas.0506580102.

Sundarrajan S, Arumugam M. 2016. Weighted gene co-expression based biomarker discovery fo r psoriasis detection. Gene, 593(1):225-234 DOI 10.1016/j.gene.2016.08.021.

Szell M, Danis J, Bata-Csorgo Z, Kemeny L. 2016. PRINS, a primate-specific long non-coding RNA, plays a role in the keratinocyte stress response and psoriasis pathogenesis. Pflügers Archiv : European Journal of Physiology 468(6):935-943 DOI 10.1007/s00424-0161803-z.

Tani H, Numajiri A, Aoki M, Umemura T, Nakazato T. 2019. Short-lived long noncoding RNAs as surrogate indicators for chemical stress in HepG2 cells and their degradation by nuclear RNases. Scientific Reports 9(1):1-10 DOI 10.1038/s41598-019-56869-y.

Tibshirani R. 1996. Regression Shrinkage and Selection Via the Lasso. Journal of the Royal Statistical Society, 58(1):267-288 DOI 10.1111/j.2517-6161.1996.tb02080.x.

Tsoi LC, Iyer MK, Stuart PE, Swindell WR., Gudjonsson JE, Tejasvi T, Elder JT. 2015. Analysis of long non-coding RNAs highlights tissue-specific expression patterns and epigenetic profiles in normal and psoriatic skin. Genome Biology 16(1):24-39 DOI 10.1186/s13059014-0570-4.

Wang P, Xue Y, Han Y, Lin L, Wu C, Xu S, Jiang Z, Xu J, Liu Q, Cao X. 2014. The STAT3binding long noncoding RNA lnc-DC controls human dendritic cell differentiation. Science, 344(6181):310-313 DOI 10.1126/science.1251456.

Wang W, Zhao Z, Yang F, Wang H, Wu F, Liang T, Zhao J. 2018. An immune-related lncRNA signature for patients with anaplastic gliomas. Journal of Neurooncology 136(2):263-271 DOI 10.1007/s11060-017-2667-6.

Watson CJ, Gupta SK, O'Connell E, Thum S, Glezeva N, Fendrich J, Gallagher J, Ledwidge M, 
518

519

520

521

522

523

524

525

526

527

528

529

530

531

532

533

534

535

536

537

538

Grote-Levi L, McDonald K, Thum T. 2015. MicroRNA signatures differentiate preserved from reduced ejection fraction heart failure. European Journal of Heart Failure 17(4): 405-415 DOI 10.1002/ejhf.244.

Xiong H, Li H, Xiao Y, Yang Q, Yang L, Chen L, Bu L, Zhang W, Zhang J, Sun Z. (2019). Long noncoding RNA MYOSLID promotes invasion and metastasis by modulating the partial epithelial-mesenchymal transition program in head and neck squamous cell carcinoma. Journal of Experimental and Clinical Cancer Research 38(1):278. DOI 10.1186/s13046-019-1254-4.

Xu, H, Jiang, Y, Xu X, Su X, Liu Y, Ma, Y, Cao X. 2019. Inducible degradation of lncRNA Sros 1 promotes IFN-gamma-mediated activation of innate immune responses by stabilizing Stat1 mRNA. Nature Immunology 20(12):1621-1630 DOI 10.1038/s41590019-0542-7.

Yadav K, Singh D, Singh MR. 2018. Protein biomarker for psoriasis: A systematic review on the ir role in the pathomechanism, diagnosis, potential targets and treatment of psoriasis. Inte rnational Journal of Biological Macromolecules 118:1796-1810.DOI 10.1016/j.ijbiomac.2 018.07.021.

Yang L, Li B, Dang E, Jin L, Fan X, Wang G. 2016. Impaired function of regulatory T cells in patients with psoriasis is mediated by phosphorylation of STAT3. Journal of Dermatological Science 81(2):85-92 DOI 10.1016/j.jdermsci.2015.11.007.

Yu G, Wang L, Han Y, He Q. 2012. clusterProfiler: an R package for comparing biological the mes among gene clusters. OMICS 16(5): 284-287 DOI 10.1089/omi.2011.0118.

Peer] reviewing PDF | (2020:06:50528:3:0:NEW 1 Feb 2021) 


\section{Figure 1}

\section{A flowchart of the GEO datasets analysis.}

Flowchart of the datasets analysis.

GSE13355 (58 psoriasis lesion samples v 64 normal skin samples )

Screening of differentially expressed genes

394 differentially expressed genes (DEGs) were extracted

immune-related genes (IRGs) were downloaded from ImmPort

76 differentially expressed immune-related genes (DEIRGs)

from DEGs were identified

Coexpression analysis

16 immune-related IncRNAs were identified

LASSO logistic regression

10 IncRNAs were identified

as diagnostic biomarkers

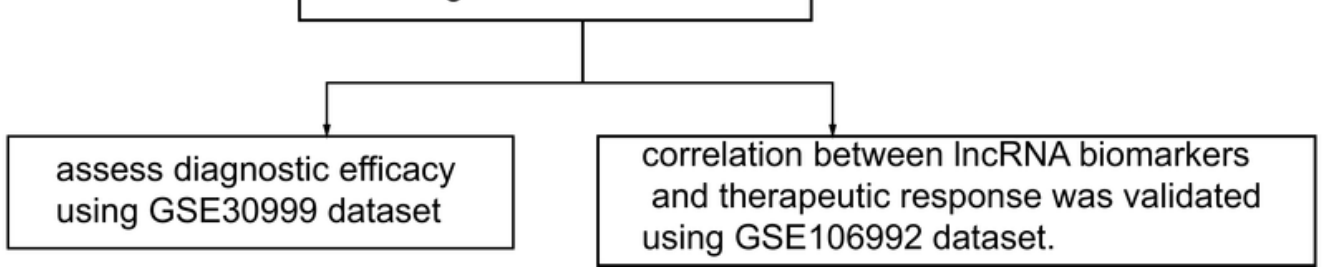


Figure 2

Density map and PCA plot before and after normalization of GSE13355 dataset.

(A, B) PCA plot before and after the batch effect removal, respectively. (C) Volcano plot of DEGs; red represents upregulated differential genes, green represents downregulated differential genes, and gray represents no differential genes

A

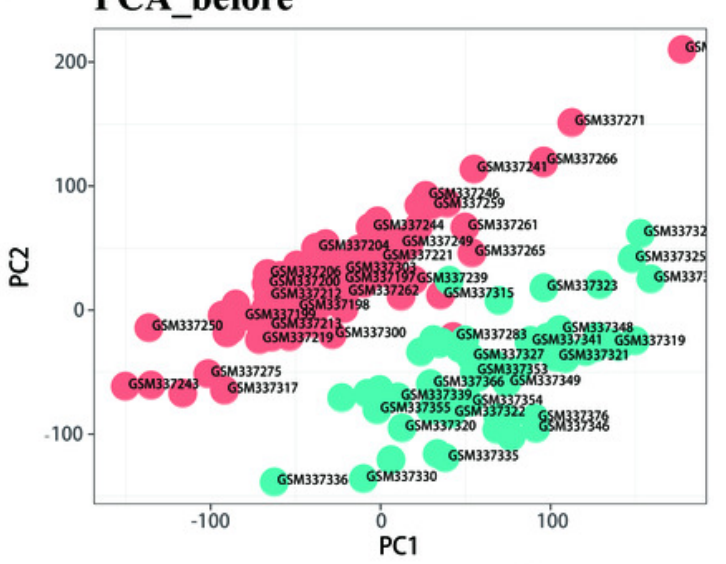

B

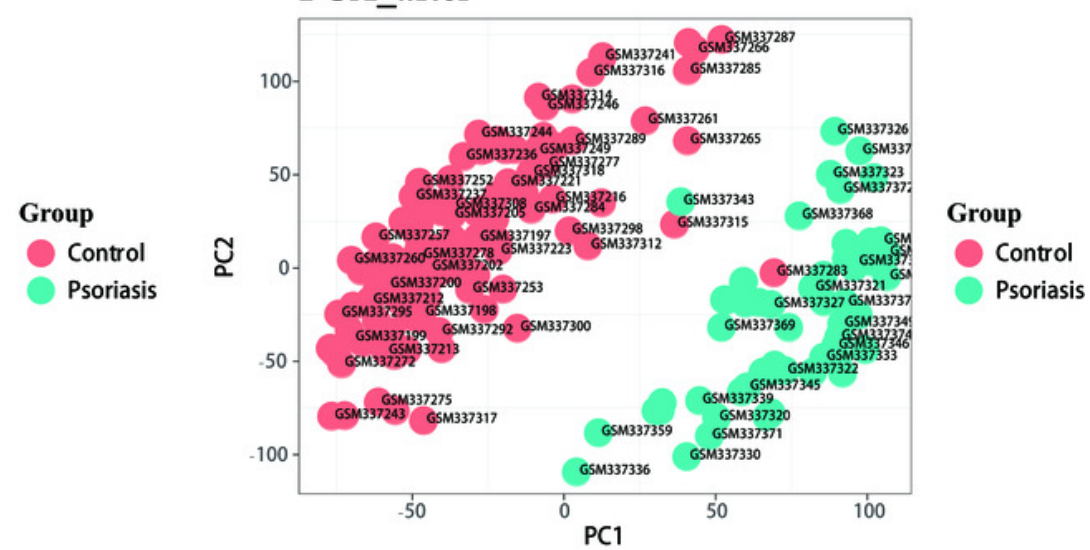

C

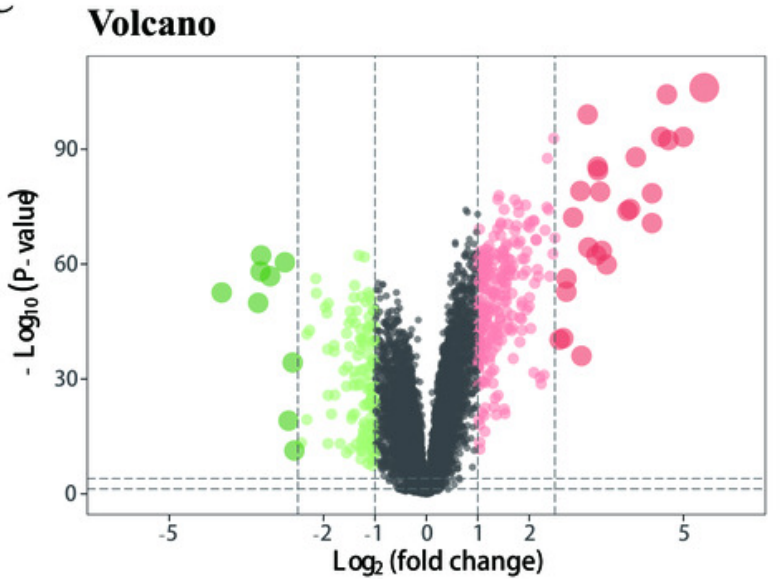




\section{Figure 3}

Functional and pathway enrichment analysis of DEGs

(A) GO enrichment analysis of biological functions. The $\mathrm{x}$-axis represents the proportion of DEGs enriched in GO team. Dot color indicates corrected P values: the brighter the red color, the smaller the corrected $\mathrm{P}$ values, and the brighter the blue color, the bigger the corrected $\mathrm{P}$ values. Dot size represents the number of enriched genes. (B) KEGG pathway analysis;

Significantly enriched KEGG pathways obtained. (C) Gene enrichment analysis; P value was calculated using Kolmogorov-Smirnov test. BP: biological process, MF: molecular function, CC: cell component. GeneRatio: the ratio of the number of genes related to this Term in the differential gene to the total number of the differential genes. 
A

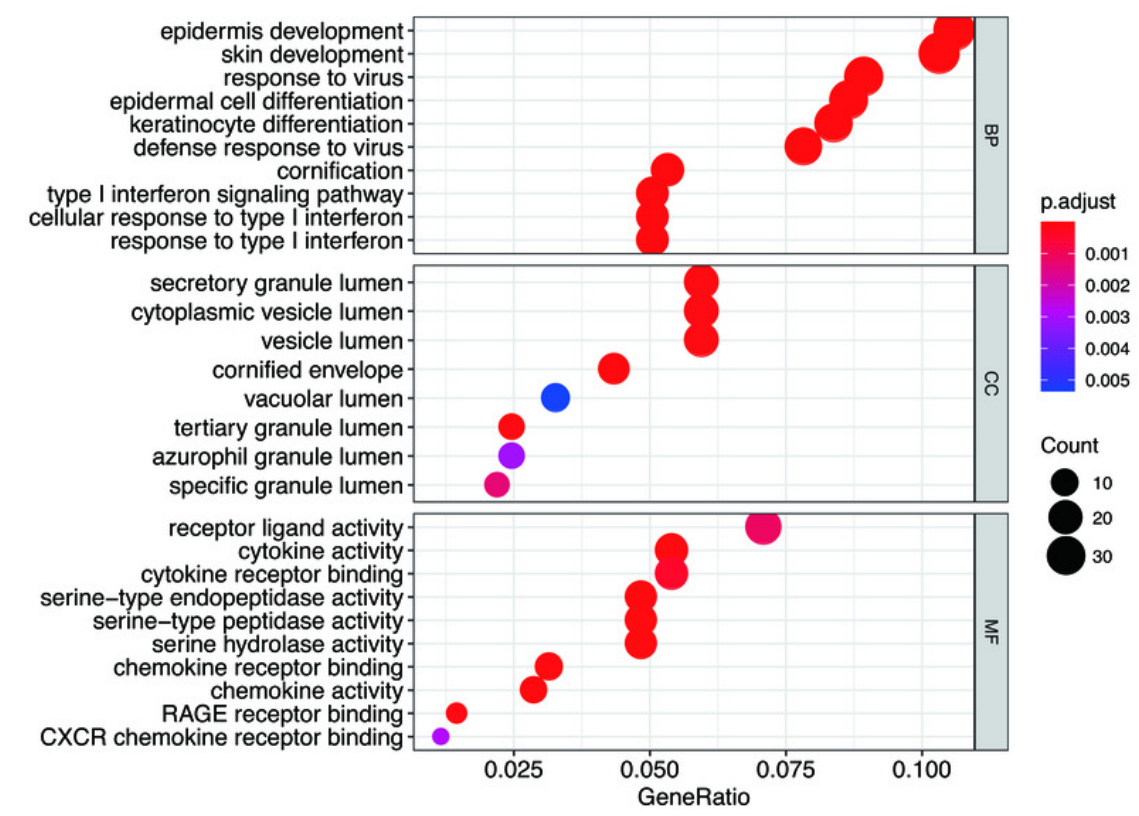

B

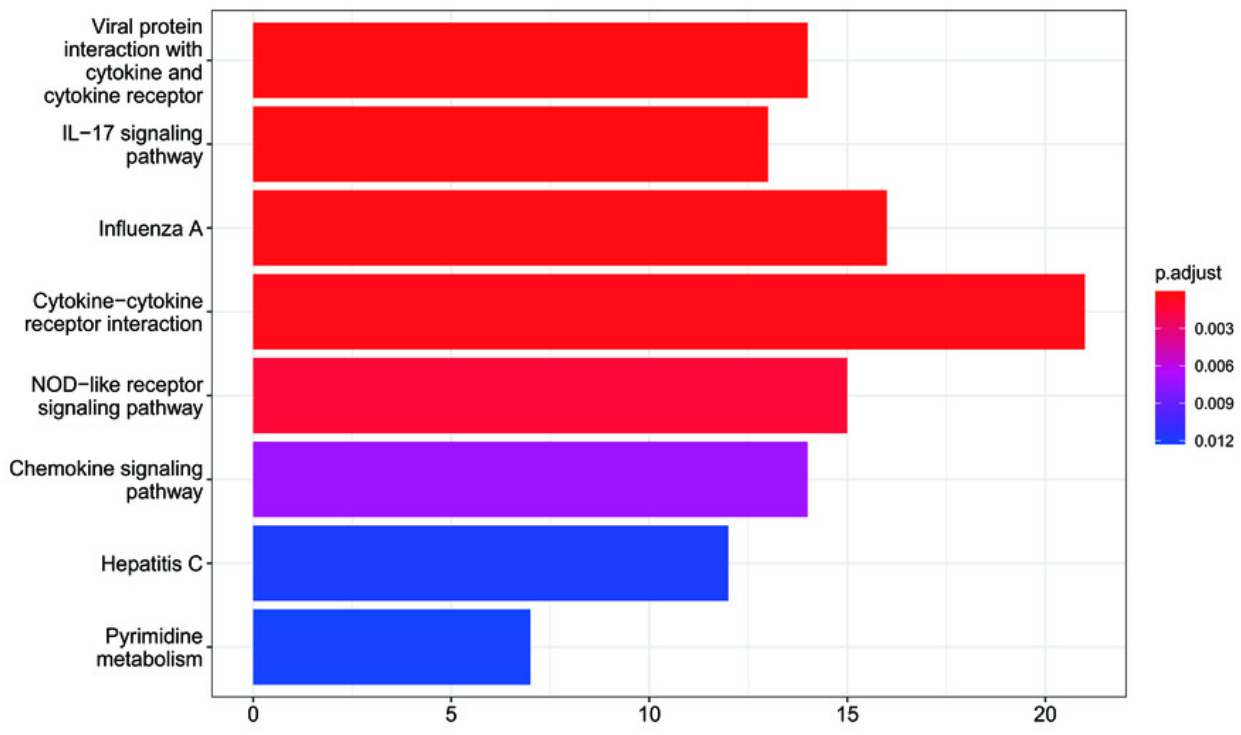

C

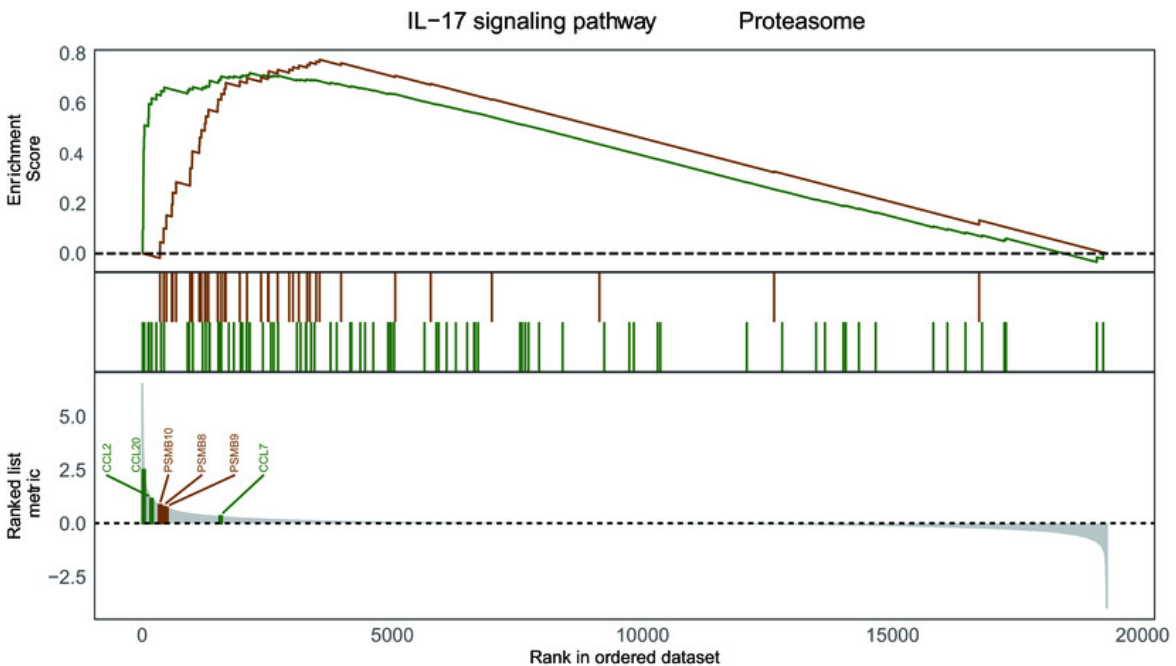

PeerJ reviewing PDF | (2020:06:50528:3:0:NEW 1 Feb 2021) 


\section{Figure 4}

Volcano plot, heatmap of differentially expressed immune-related genes (DEIRGs), and IncRNA functional annotation.

(A) Volcano plot of DEIRGs; red represents upregulated differential genes, green represents downregulated differential genes, and gray represents no differential genes. (B) GO analysis of immune-related IncRNA target genes. (C) KEGG analysis of immune-related IncRNA target genes. The x-axis represents the proportion of DEGs enriched in GO team. Dot color indicates corrected $\mathrm{P}$ values: the brighter the red color, the smaller the corrected $\mathrm{P}$ values; the brighter the blue color, the bigger the corrected $P$ values. Dot size represents the number of enriched genes 

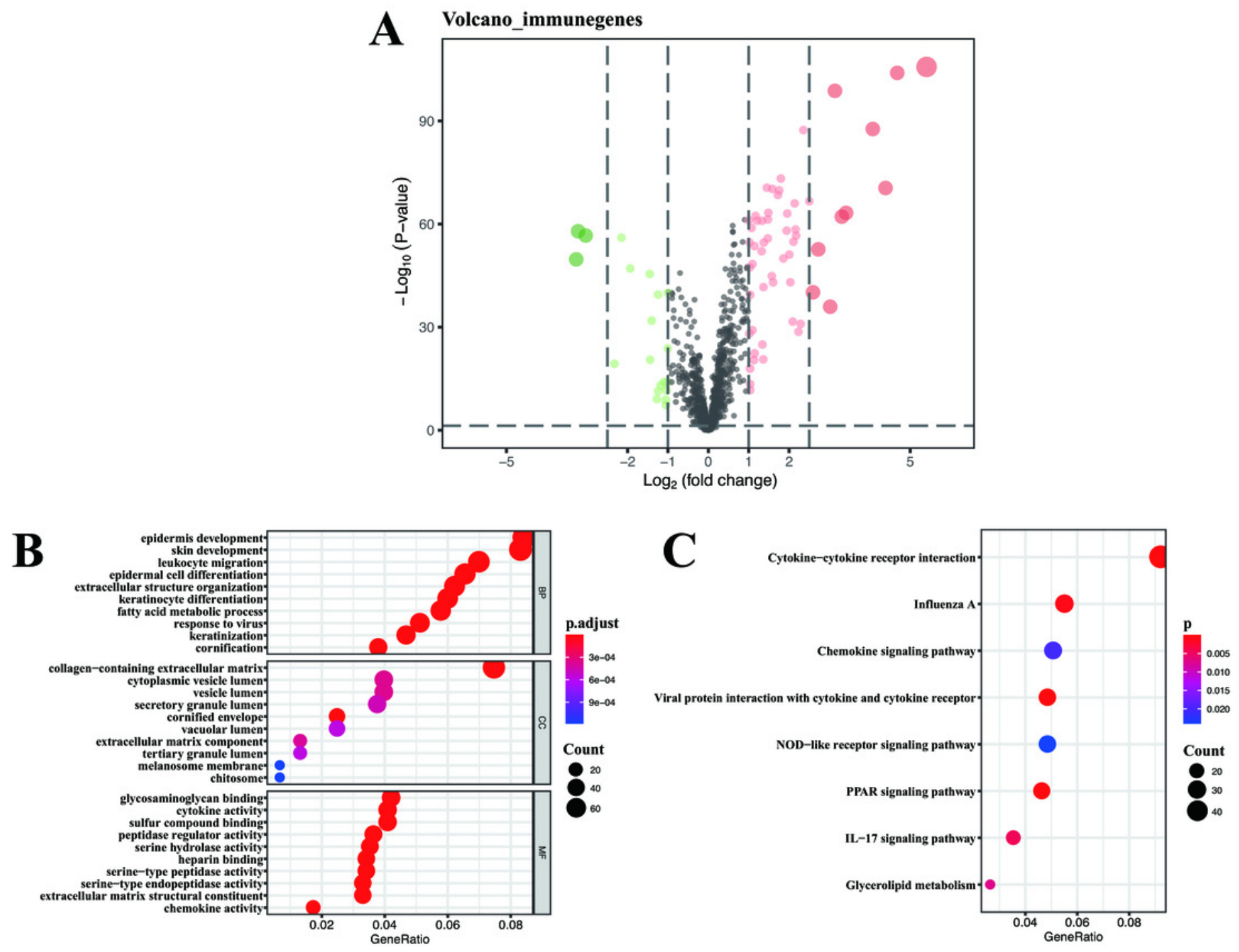
Figure 5

Diagnostic biomarker identification and validation.

(A) 10 IncRNAs were identified as potential diagnostic biomarkers for psoriasis by LASSO logistic regression algorithms with 10-fold cross-validation. (B, C) ROC curve for diagnostic biomarker in test dataset
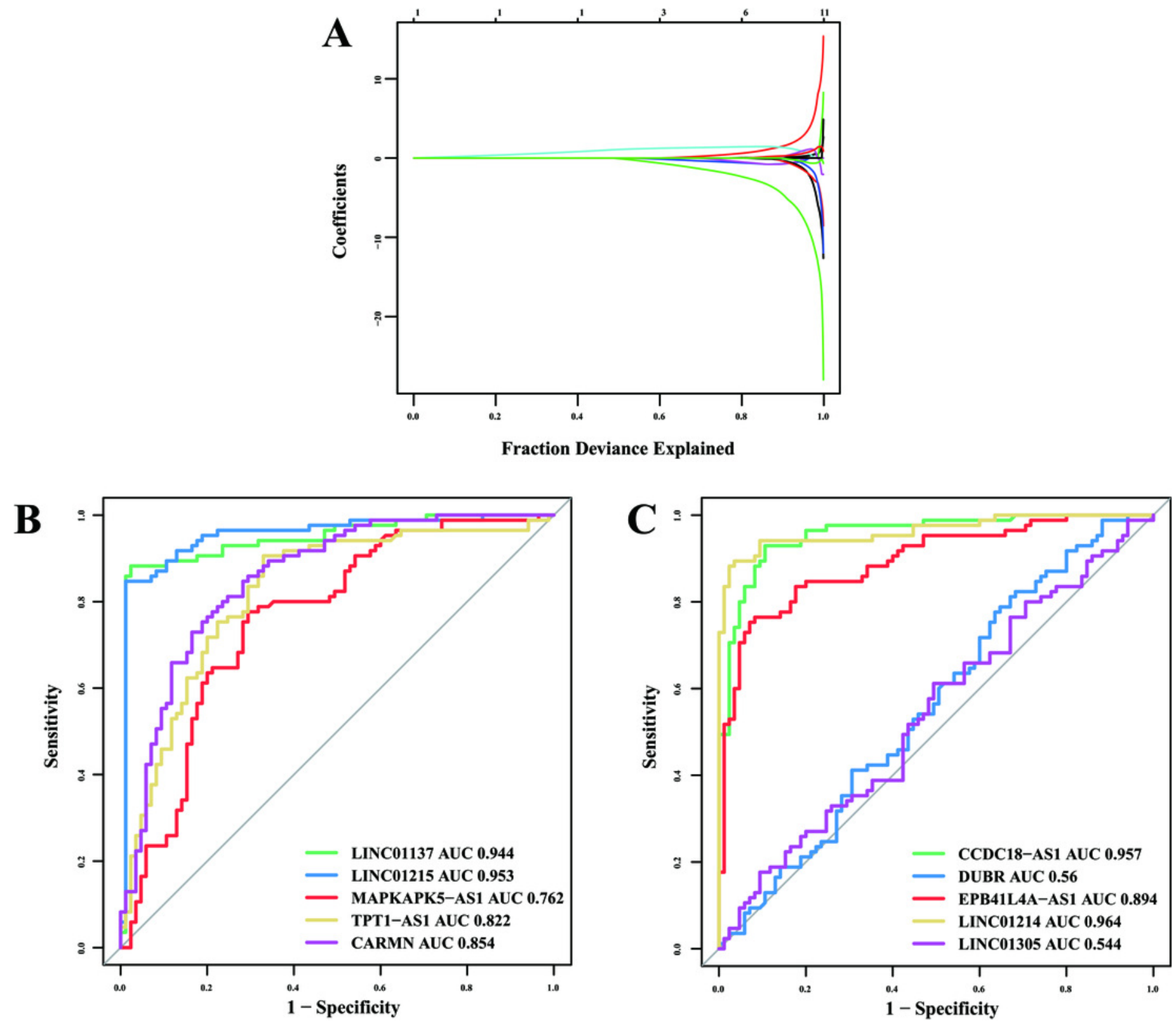
Figure 6

Heatmap of diagnostic biomarker and biologics response correlation analysis.

Heatmap of diagnostic biomarker and biologics response correlation analysis. ****P $<.00001$

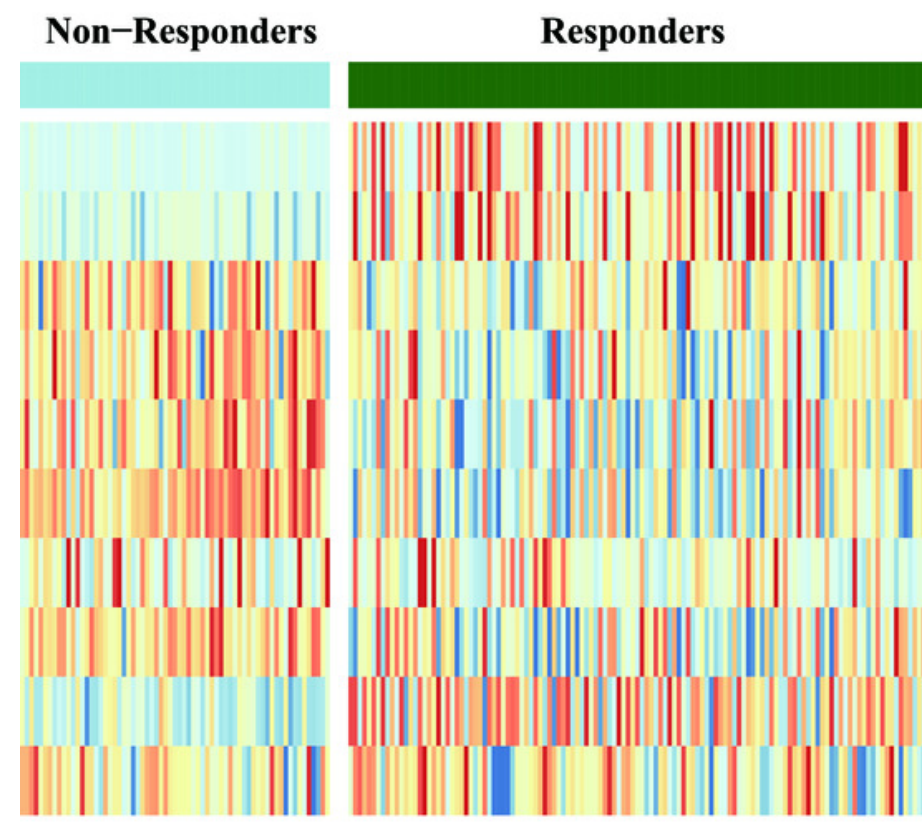

Subtype

LINC01137****

LINC01215****

MAPKAPK5.AS1****

TPT1.AS1****

CARMN****

CCDC18.AS1****

DUBR

EPB41L4A.AS1****

LINC01214****

LINC01305
P-value

4.02e-12

$1.90 \mathrm{e}^{-11}$

$3.33 \mathrm{e}-04$

$3.43 \mathrm{e}-06$

$1.04 \mathrm{e}^{-05}$

$4.02 \mathrm{e}^{-12}$

$8.59 \mathrm{e}-01$

8.81e-06

$1.69 \mathrm{e}-10$

$7.61 e^{-01}$
2

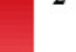

1

0

$-1$ 


\section{Figure 7}

Assessment and visualization of immune cell infiltration.

(A) Heatmap of differentially infiltration of immune cells between psoriasis group and control group. (B) PCA plots show the clustering of immune cell infiltration in the psoriasis group and control group. (C) Heatmap of correlation of infiltration between 22 immune cells. Blue indicates positive correlation, whereas red indicates negative correlation; the darker the color, the stronger the correlation. 


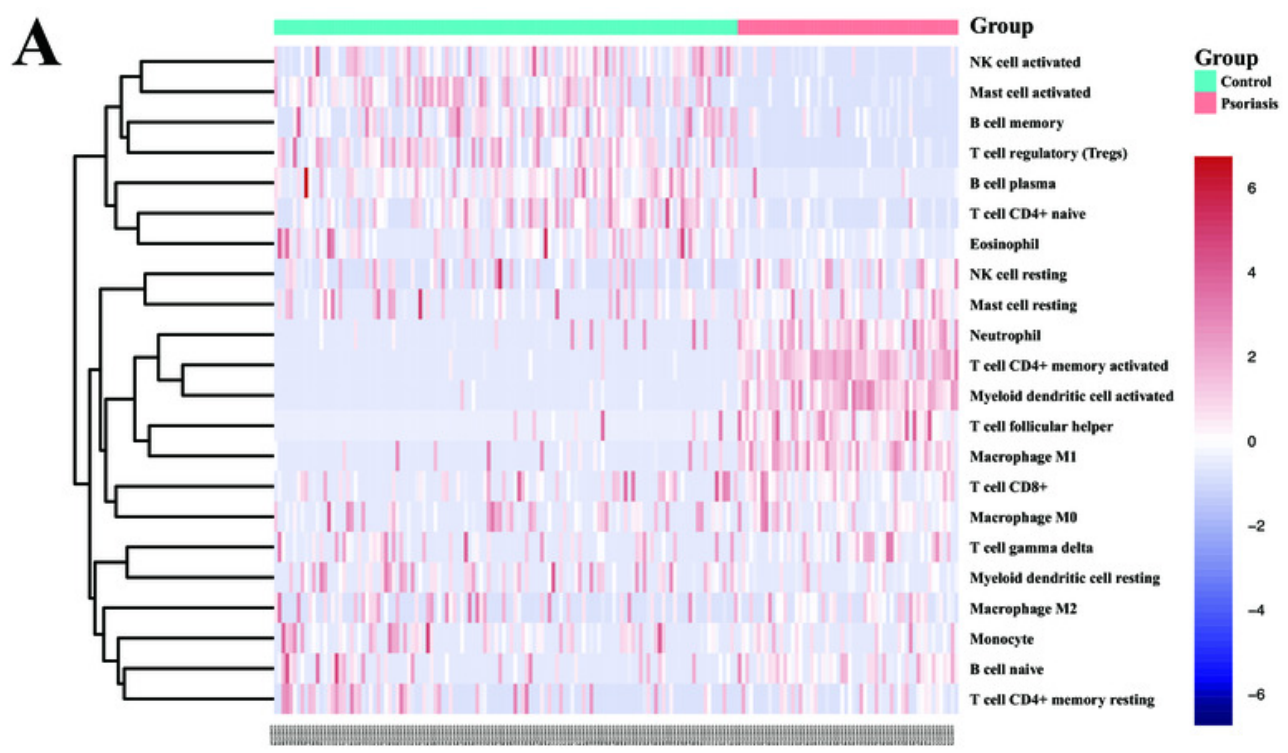

B

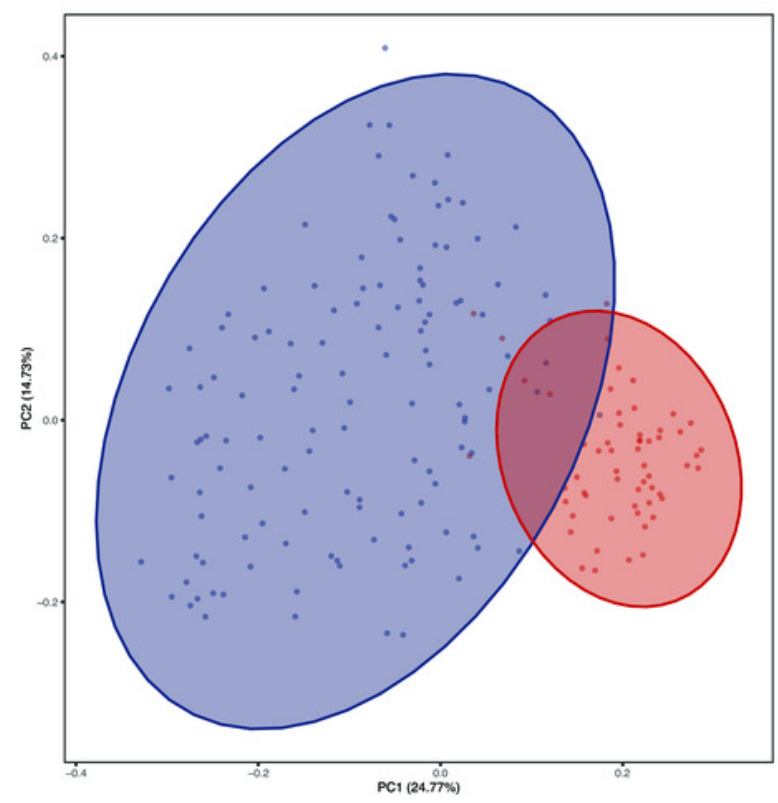

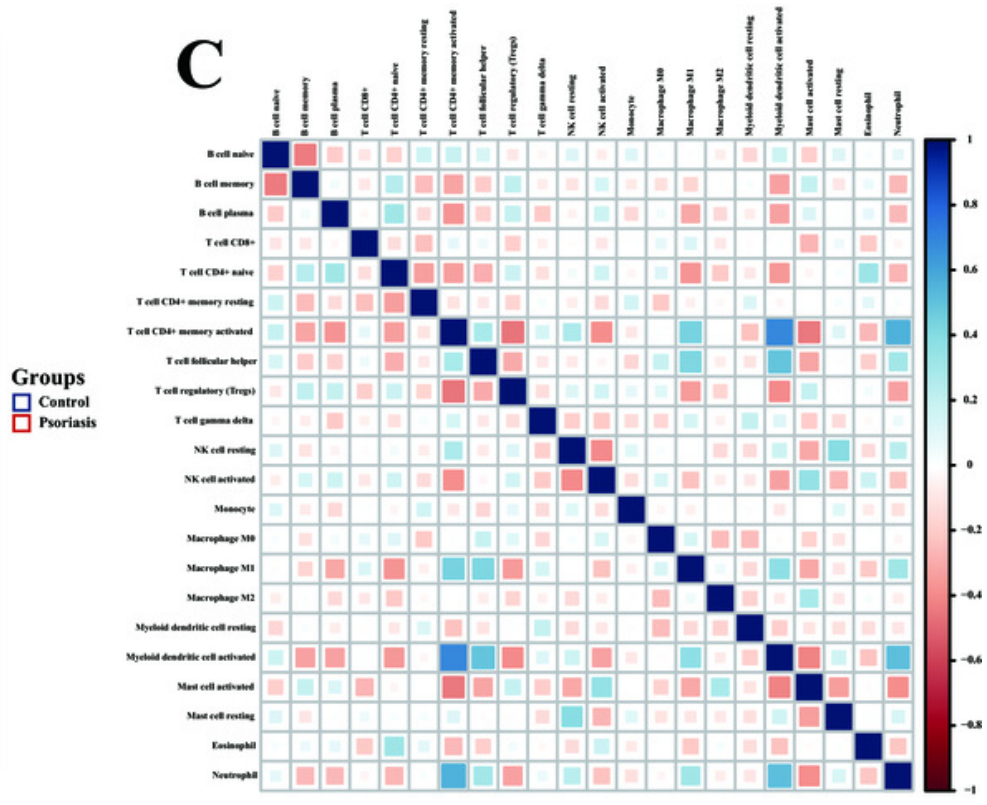




\section{Figure 8}

Visualization of immune cell infiltration and correlation analysis with diagnostic biomarker.

(A) Violin plots of the proportion of infiltration of 22 immune cells. Red represents the psoriasis group, and blue represents the control group. (B) Interaction plots of infiltration of 22 immune cells. The size of the circle represents interaction strength; the bigger the circle, the stronger the interaction. (C) The correlation analysis of infiltration between 22 immune cells and immune-related IncRNA diagnostic biomarkers; red represents positive correlation, and blue represents negative correlation. 
$\mathbf{A}$

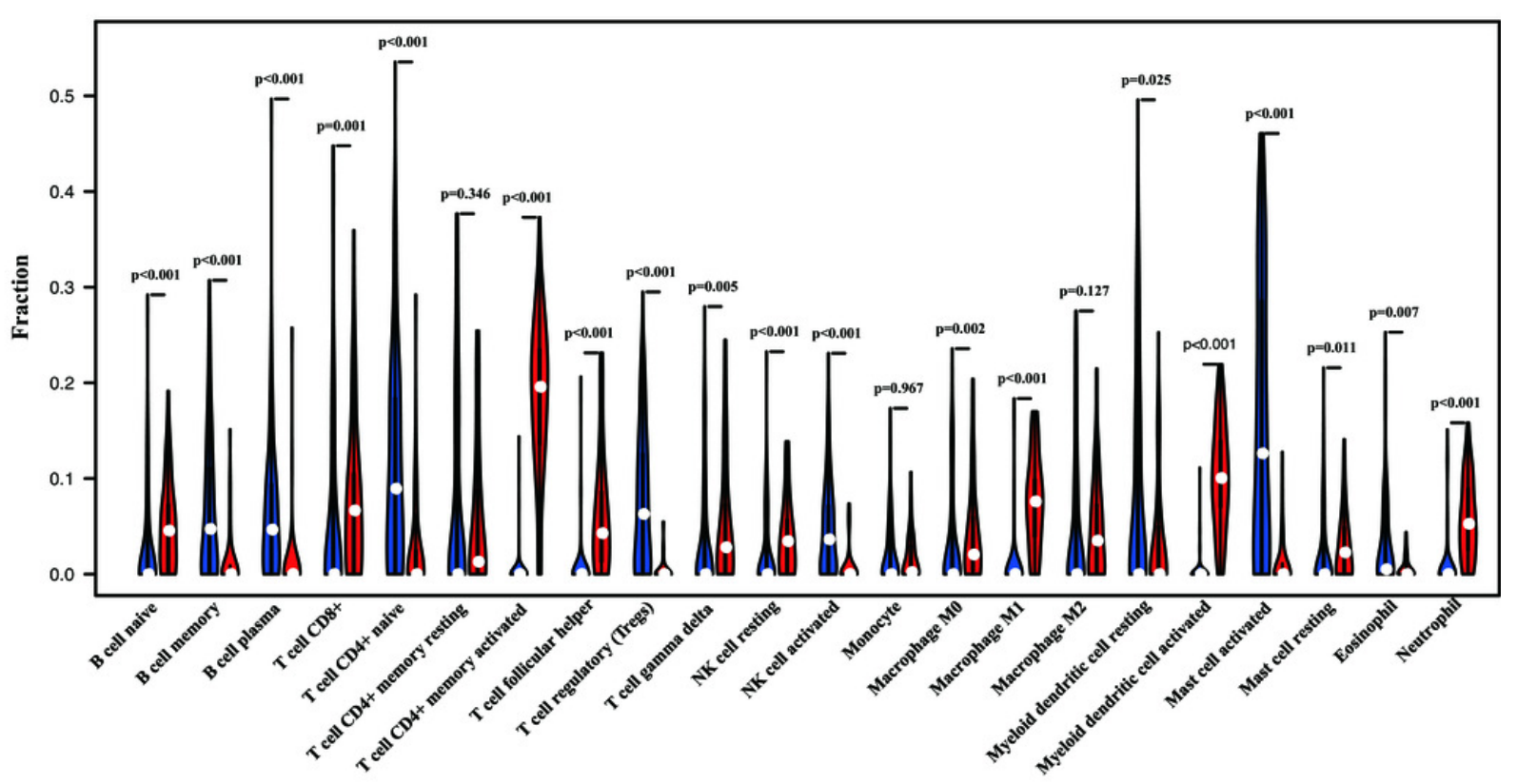

B

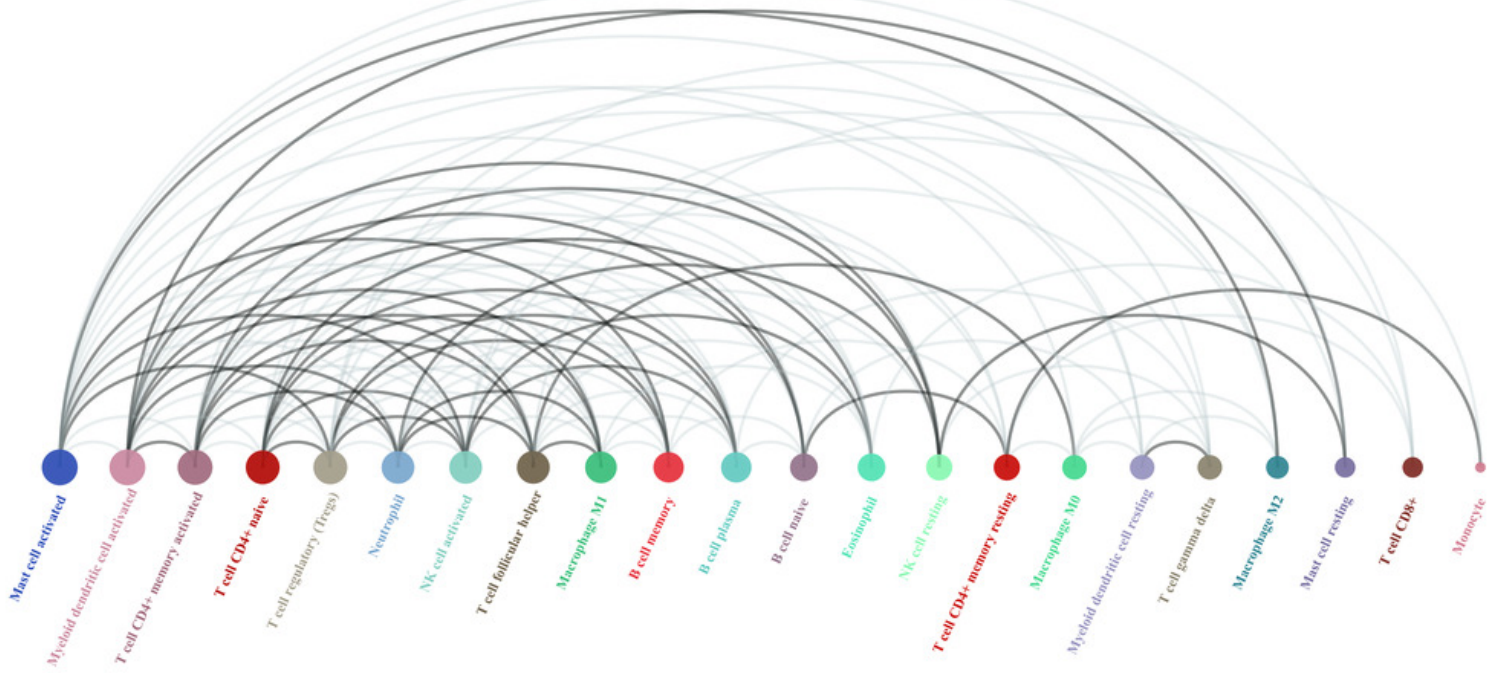

C
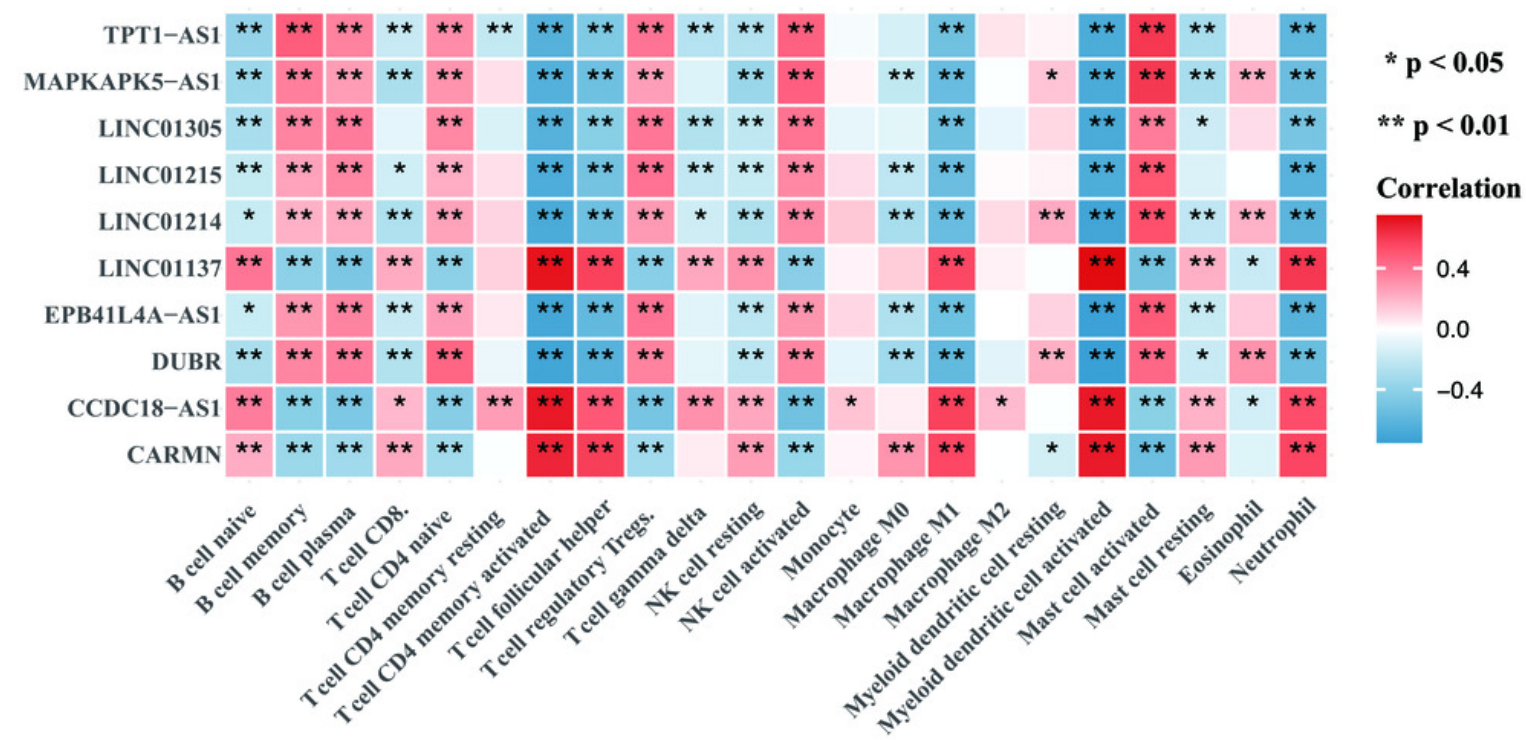


\section{Table $\mathbf{1}$ (on next page)}

The top 10 differentially expressed genes (up regulated)

$\log \mathrm{FC}$ and $\mathrm{p}$ value of these top 10 up regulated differentially expressed genes were presented in table 


\begin{tabular}{llll}
\hline Gene symbol & $\operatorname{logFC}$ & P-value & adj P-value \\
\hline SERPINB4 & 6.553829 & $1.98 \mathrm{E}-82$ & $3.44 \mathrm{E}-79$ \\
PI3 & 5.404915 & $8.91 \mathrm{E}-107$ & $1.86 \mathrm{E}-102$ \\
TCN1 & 4.999575 & $6.35 \mathrm{E}-94$ & $2.65 \mathrm{E}-90$ \\
SPRR2C & 4.709046 & $4.24 \mathrm{E}-93$ & $1.26 \mathrm{E}-89$ \\
S100A12 & 4.677867 & $4.94 \mathrm{E}-105$ & $5.15 \mathrm{E}-101$ \\
AKR1B10 & 4.568268 & $5.91 \mathrm{E}-94$ & $2.65 \mathrm{E}-90$ \\
SERPINB3 & 4.389195 & $3.26 \mathrm{E}-79$ & $4.53 \mathrm{E}-76$ \\
S100A9 & 4.388357 & $1.97 \mathrm{E}-71$ & $1.18 \mathrm{E}-68$ \\
IL36G & 4.072299 & $1.21 \mathrm{E}-88$ & $3.16 \mathrm{E}-85$ \\
C10orf99 & 3.962806 & $4.10 \mathrm{E}-75$ & $3.88 \mathrm{E}-72$ \\
\hline
\end{tabular}

1 


\section{Table 2 (on next page)}

The top 10 differentially expressed genes (down regulated)

$\log \mathrm{FC}$ and $\mathrm{p}$ value of these top 10 down regulated differentially expressed genes were presented in table 


\begin{tabular}{llll}
\hline Gene symbol & $\operatorname{logFC}$ & P-value & adj P-value \\
\hline WIF1 & -3.98135 & $2.64 \mathrm{E}-53$ & $2.54 \mathrm{E}-51$ \\
BTC & -3.27259 & $1.33 \mathrm{E}-50$ & $1.07 \mathrm{E}-48$ \\
CCL27 & -3.22479 & $8.40 \mathrm{E}-59$ & $1.33 \mathrm{E}-56$ \\
KRT77 & -3.21433 & $5.75 \mathrm{E}-63$ & $1.43 \mathrm{E}-60$ \\
IL37 & -3.03789 & $1.49 \mathrm{E}-57$ & $2.11 \mathrm{E}-55$ \\
C5orf46 & -2.75129 & $3.38 \mathrm{E}-61$ & $6.77 \mathrm{E}-59$ \\
THRSP & -2.6802 & $7.85 \mathrm{E}-20$ & $6.04 \mathrm{E}-19$ \\
MSMB & -2.59999 & $5.13 \mathrm{E}-35$ & $1.19 \mathrm{E}-33$ \\
PM20D1 & -2.56708 & $4.88 \mathrm{E}-12$ & $2.09 \mathrm{E}-11$ \\
ELOVL3 & -2.43197 & $4.72 \mathrm{E}-14$ & $2.35 \mathrm{E}-13$ \\
\hline
\end{tabular}

1 


\section{Table 3(on next page)}

The details of 16 immune-related IncRNAs

The gene symbol, gene type, description, location and phenotypes of the 16 immune-related IncRNAs. 
1 Table 3. The details of the 16 immune-related lncRNAs

\begin{tabular}{|c|c|c|c|c|c|}
\hline $\begin{array}{l}\text { Gene } \\
\text { symbol }\end{array}$ & Gene type & $\begin{array}{l}\text { Correlation } \\
\text { coefficients }\end{array}$ & $\begin{array}{l}\mathrm{P} \\
\text { value }\end{array}$ & Location & Phenotypes \\
\hline LINC01214 & LncRNA & 0.832 & $2.13 \mathrm{E}-47$ & $\begin{array}{l}\text { Chr3:150,265,407- } \\
150,296,65\end{array}$ & No report \\
\hline LINC01215 & LncRNA & 0.842 & $1.09 \mathrm{E}-49$ & $\begin{array}{l}\text { Chr3: } 108,125,821- \\
108,138,610\end{array}$ & No report \\
\hline LINC01137 & LncRNA & 0.861 & $4.43 \mathrm{E}-54$ & $\begin{array}{l}\text { Chr1: } 37,350,934- \\
37,474,411\end{array}$ & $\begin{array}{l}\text { Vaccinia virus } \\
\text { infection }\end{array}$ \\
\hline LINC01305 & LncRNA & 0.710 & $5.72 \mathrm{E}-29$ & $\begin{array}{l}\text { Chr2: } 174,326,027- \\
174,330,643\end{array}$ & $\begin{array}{l}\text { epithelial- } \\
\text { mesenchymal } \\
\text { transition }\end{array}$ \\
\hline CARMN & LncRNA & 0.779 & $5.65 \mathrm{E}-38$ & $\begin{array}{l}\text { Chr5: } 149,406,689- \\
149,432,835\end{array}$ & $\begin{array}{l}\text { Vaccinia virus } \\
\text { infection }\end{array}$ \\
\hline CCDC18-AS1 & LncRNA & 0.724 & $1.56 \mathrm{E}-30$ & $\begin{array}{l}\text { Chr1: 93,262,186- } \\
93,346,025\end{array}$ & No report \\
\hline DUBR & LncRNA & 0.754 & $2.53 \mathrm{E}-34$ & $\begin{array}{l}\text { Chr3: } 107,220,744- \\
107,348,464\end{array}$ & No report \\
\hline EPB41L4A-AS & LncRNA & 0.772 & 7.94E-37 & $\begin{array}{l}\text { Chr5: } 112,160,526- \\
112,164,818\end{array}$ & $\begin{array}{l}\text { metabolic } \\
\text { reprogramming }\end{array}$ \\
\hline $\begin{array}{l}\text { MAPKAPK5- } \\
\text { AS1 }\end{array}$ & LncRNA & 0.795 & $2.03 \mathrm{E}-40$ & $\begin{array}{l}\text { Chr12:111,839,764- } \\
111,842,902\end{array}$ & tumorigenesis \\
\hline TPT1-AS1 & LncRNA & 0.766 & $5.65 \mathrm{E}-36$ & $\begin{array}{l}\text { Chr13: 45,341,345- } \\
45,417,975\end{array}$ & tumor promotion \\
\hline PGM5-AS1 & LncRNA & 0.854 & $1.81 \mathrm{E}-52$ & $\begin{array}{l}\text { Chr9: 68,353,614- } \\
68,357,893\end{array}$ & $\begin{array}{l}\text { tumor } \\
\text { suppression }\end{array}$ \\
\hline $\begin{array}{l}\text { SH3PXD2A- } \\
\text { AS1 }\end{array}$ & LncRNA & 0.909 & $1.50 \mathrm{E}-69$ & $\begin{array}{l}\text { Chr10:103,745,966- } \\
103,755,423\end{array}$ & tumor promotion \\
\hline LINC00173 & LncRNA & 0.745 & $4.26 \mathrm{E}-33$ & $\begin{array}{l}\text { Chr12:116,533,422- } \\
116,536,518\end{array}$ & chemoresistance \\
\hline LINC00518 & LncRNA & 0.731 & $2.61 \mathrm{E}-31$ & $\begin{array}{l}\text { Chr6: } 10,429,255- \\
10,435,015\end{array}$ & $\begin{array}{l}\text { tumor promotion, } \\
\text { chemoresistance }\end{array}$ \\
\hline LINC00526 & LncRNA & 0.755 & $1.68 \mathrm{E}-34$ & $\begin{array}{l}\text { Chr18: 5,236,724- } \\
5,238,598\end{array}$ & $\begin{array}{l}\text { tumor } \\
\text { suppression }\end{array}$ \\
\hline EMX2OS & LncRNA & 0.811 & $2.36 \mathrm{E}-43$ & $\begin{array}{l}\text { Chr2: } 117484293- \\
117545068\end{array}$ & No report \\
\hline
\end{tabular}

2 


\section{Table 4 (on next page)}

The details of the AUC and $95 \% \mathrm{Cl}$ of AUC of the 10 immune-related IncRNAs

The gene symbol, AUC and $95 \% \mathrm{Cl}$ of AUC of the 10 immune-related IncRNAs. 


\begin{tabular}{lll}
\hline Gene symbol & AUC & $95 \%$ CI of AUC \\
\hline LINC01137 & 0.944 & $0.907-0.982$ \\
LINC01215 & 0.953 & $0.919-0.987$ \\
MAPKAPK5-AS1 & 0.762 & $0.688-0.835$ \\
TPT1-AS1 & 0.822 & $0.758-0.887$ \\
CARMN & 0.854 & $0.797-0.911$ \\
CCDC18-AS1 & 0.957 & $0.929-0.985$ \\
DUBR & 0.56 & $0.474-0.647$ \\
EPB41L4A-AS1 & 0.894 & $0.846-0.942$ \\
LINC01214 & 0.964 & $0.937-0.991$ \\
LINC01305 & 0.544 & $0.457-0.631$ \\
\hline
\end{tabular}

1 